\title{
Lipid-Based Delivery System for Topical Phenytoin
}

\author{
Siang Yin Lee ${ }^{1,2^{*}}$, Yeong Yaw Pung ${ }^{1}$, Boon Kuang Khor ${ }^{1}$, Wei En Kong ${ }^{1}$, Cai Ting $\operatorname{Tan}^{1}$, Siew Yong Teo \\ ${ }^{1}$ Department of Pharmaceutical Chemistry, School of Pharmacy, International Medical University, No. 126, Jalan Jalil Perkasa 19, Bukit Jalil, 57000 Kuala \\ Lumpur, Malaysia. ${ }^{2}$ Unit Colloids and Interface Science (CISC), Centre of Excellence (COE), RRIM Sungai Buloh research station, Malaysian Rubber \\ Board (MRB), 47000 Sungai Buloh, Selangor, Malaysia. ${ }^{3}$ School of Postgraduate Studies and Research, International Medical University, No. 126, Jalan \\ Jalil Perkasa 19, Bukit Jalil, 57000 Kuala Lumpur, Malaysia.
}

\begin{tabular}{l} 
ARTICLE INFO \\
\hline Article history: \\
Received on: $12 / 06 / 2016$ \\
Revised on: 10/07/2016 \\
Accepted on: 22/08/2016 \\
Available online: 29/11/2016 \\
\hline Key words: \\
Cream-gel, lipid, macro- \\
emulgel, nano-emulgel, \\
phenytoin, topical.
\end{tabular}

\begin{abstract}
The wound healing effect of topical phenytoin has been reported but its effective delivery system is still underresearched. The present work aimed to formulate topical lipid-based gels for effective delivery of phenytoin. Three lipid-based emulsions with different droplet sizes were prepared using coconut kernel oil (CKO) and soybean oil (SBO) blends as an oil phase. Stable lipid-based emulsion with $100 \%$ emulsion stability index (ESI) over seven heating/cooling cycles could only be formed when the oil droplet satisfied the nano size criterion, as demonstrated by nano-emulsion. The stability of macro-emulsion and cream could be enhanced by the addition of Aristoflex ammonium acryloyldimethyl-taurate/VP (AVC) copolymer as the gelling agent. These gels, designated as nano-emulgel, macro-emulgel and cream-gel, were subsequently loaded with phenytoin. All gels exhibited non-Newtonian and shear-thinning flow behavior, with high yield stress ranged 18.8 -51.5 Pa. The in vitro drug release profiles of all formulations followed the first-order kinetic model, with $\mathrm{R}^{2}>0.95$. It was found that lipid nano-emulgel demonstrated the highest release rate of phenytoin, with $93.12 \%$ drug released in 12 hours, followed by cream-gel with $56.42 \%$ and macro-emulgel with $51.51 \%$. CKO/SBO nano-emulgel was identified as the most suitable lipid topical gel formulation for effective delivery of phenytoin.
\end{abstract}

\section{INTRODUCTION}

Phenytoin was first introduced in 1938 as an antiepileptic agent as the first-line therapeutic approach for various generalized convulsive disorders. Many studies showed that $50 \%$ of the patients receiving long-term oral phenytoin treatment were presented with a side effect of gingival hyperplasia (Shaw et al., 2007). This apparent stimulatory effect on the connective tissues suggested that phenytoin has wound healing effect (Simsek et al., 2014; Spaia, 2004). The clinical effects of topical phenytoin on wound healing have been systematically reviewed by Shaw et al. (Shaw et al., 2007). Various clinical trials have reported an improved healing rate of cutaneous wounds, decubitus ulcers, diabetic foot ulcers, burn wounds, traumatic wounds and abscesses with reduced inflammation and edema (Bahmani et al., 2011; Fonseka et al., 2010; Hokkam et al., 2011; Rashidi et al., 2012; Rhodes et al., 2001). To-date, despite the extensive studies was done on the

* Corresponding Author

Siang Yin Lee, Research Officer of Malaysian Rubber Board

Email: leesiangyin@lgm.gov.my wound healing effect of topical phenytoin, it is still used as offlabel medications. Its widespread use in clinical settings was restricted by the fact that the delivery method of topical phenytoin has not been adequately studied and thus the best formulation to deliver the drug is not known (Smith, 2010). Rhodes et al. reported that majority of studies merely stated that a uniform, thin layer of phenytoin powder was applied to the wound surface (Rhodes et al., 2001). However, powders are difficult to be held on the skin in addition to the difficulty in putting the recommended amount of the powder on the affected area. Topical phenytoin sodium suspensions ( 2 and $4 \% \mathrm{w} / \mathrm{w}$ ) had been using to apply directly to the wounds. Nevertheless, the efficiencies of these formulations remained controversial due to the low contact period of phenytoin over the wounds and difficulty for even application (Rhodes et al., 2006; Almousilly et al., 2012; Fonseka et al., 2010). Thus, a novel drug carrier is needed to be developed for effective control and improved drug delivery of topical phenytoin. For decades, lipids have gained much interest as the promising pharmaceutical carriers for delivery of drugs and enhancing the bioavailability of poorly water-solubility drugs, (Almousilly et al., 2012; Pouton, 2006; Shrestha et al., 2014). 
These lipid-based drug delivery system (LBDDS) have been widely investigated to encapsulate or solubilize the anticancer, antibacterial and antiviral drugs (Jones et al., 2004; Mehanna et al., 2012; Yadav et al., 2013). The key advantages of lipid-based formulations are (1) enhanced drug bioavailability, (2) increased drug solubility and (3) negligible toxicity. Lipid-based formulations may include oil solutions or suspensions, emulsions, self-micro or self-nano emulsifying drug delivery systems (SMEDDS/ SNEDDS) (Čerpnjak et al., 2013). An appropriate selection of lipid phase, formulation strategies, and rational delivery system design can lead to the success of LBDDS formulations (Dahan and Hoffman, 2008).

The chosen lipid phase in this study was a blend of coconut kernel oil (CKO) and soya bean oil (SBO). CKO is saturated lipid with medium-chain triglycerides (MCTs) extracted from the kernel of coconuts harvested from the coconut palm (Cocos nucifera); whereas SBO is long-chain triglycerides (LCTs) obtained from soybeans (Glycina maxima). According to reported literature data, MCTs exhibit better self-dispersing ability, greater self-emulsification ability and higher chemical stability compared to LCT. However, LCT has higher solubilizing capacity than MCT for highly lipophilic drugs (Prajapati et al., 2012; Bolko et al., 2013). Hence, mixed lipid phase composed of both MCT and LCT was expected to have better physicochemical properties in preparing the LBDDS formulations for this study.

Herein, the present work describes the formulation and characterization of the LBDDS for topical application of phenytoin. Lipids were formulated into nano-emulgel and the efficacy of the in vitro release of phenytoin from this formulation was compared with those of macro-emulgel and cream-gel. The storage stability, rheological properties and release kinetics of each formulation system were evaluated to assess their suitability as drug delivery formulations for topical phenytoin.

\section{MATERIALS AND METHODS}

\section{Chemicals}

Tween 80 and sodium benzoate were purchased from Fisher Scientific Sdn. Bhd. (Selangor, Malaysia). Aristoflex ammonium acryloyldimethyl-taurate/VP (Aristoflex ${ }^{\circledR}$ AVC) copolymer was purchased from Cosmatrixx Sdn. Bhd. (Selangor, Malaysia). Phenytoin was purchased from Sigma-Aldrich Sdn. Bhd. (Kuala Lumpur, Malaysia). Coconut kernel oil (CKO) and soybean oil (SBO) were gifted from MOI Foods Sdn. Bhd. (Selangor, Malaysia). All chemicals were of reagent grade and used as received.

\section{Formulation of lipid-based delivery system}

Three types of topical gels (designated as nano-emulgel, macro-emulgel and cream-gel) were formulated from $\mathrm{CKO} / \mathrm{SBO}$ blend as an oil phase, Tween 80 as the non-ionic surfactant, distilled water containing $0.1 \% \mathrm{w} / \mathrm{w}$ sodium benzoate and $1 \% \mathrm{w} / \mathrm{w}$ Aristoflex ${ }^{\circledR}$ AVC as a gelling agent. To prepare a nano-emulgel, nano-emulsion was first prepared from the mixture of oil, surfactant and water using phase inversion temperature (PIT) method described by Shinoda (Shinoda et al., 1969). The mixture was stirred in a vessel continuously using a magnetic stirrer at 250 rpm and warmed up gradually using a water bath. The change of conductivity was measured as a function of temperature using a conductivity meter. The PIT of each formulation was determined using Oakton Con II conductivity meter. After the phase inversion point had reached, the emulsion was rapidly cooled by immersion in an ice bath with continuous stirring. The resultant emulsion was blended with a gelling agent and homogenized at room temperature at 10,000 rpm for 10 minutes. The macro-emulgel was prepared by first mixing the oil and surfactant into a vessel agitated with a magnetic stirrer at $250 \mathrm{rpm}$. The mixture was slowly titrated with water at room temperature until a concentration of $90 \% \mathrm{w} / \mathrm{w}$ was reached. The gelling agent was then added to the resultant emulsion and homogenized at room temperature at $10,000 \mathrm{rpm}$ for 10 minutes. The cream gel was prepared by first mixing the oil and surfactant into a vessel with constant stirring at room temperature. The gelling agent was then sprinkled into the mixture. Subsequently, water in approximately equal proportions with oil was added to the mixture and homogenized at 10,000 rpm for 10 minutes.

Phenytoin-loaded topical gels were formulated by first mixing $0.20 \% \mathrm{w} / \mathrm{w}$ of phenytoin into the mixture of oil and surfactant at room temperature. The mixture was vortexed thoroughly at $1400 \mathrm{rpm}$ until a clear dispersion was formed, indicating the completion of drug solubilization. The mixture followed the steps as described above to obtain drug-loaded nanoemulgel, macro-emulgel and cream gel formulations.

\section{Physico-chemical evaluation of lipid emulsions}

Emulsions were subjected to droplet size determinations. The measurements of droplet size were performed using a Zetasizer ZEN 3600 (Malvern, Worcestershire, UK) at room temperature. Droplet size measurements were determined using dynamic light scattering (DLS) and calibrated using polystyrene latex standard. The $\mathrm{pH}$ of gel formulations was assessed using Mettler Toledo S220 Seven Compact pH meter. All measurements were performed in triplicate.

\section{Emulsion stability index}

All emulsions were subjected to heating/cooling test for seven cycles, where storage temperature was changed between $4^{\circ} \mathrm{C}$ and $40^{\circ} \mathrm{C}$ in an incubator (Heraeus, Hanau, Germany) for the duration of 14 days. Emulsion stability index (ESI) of each emulsion was measured to evaluate the degree of phase separation using the equation as shown below.

$$
\mathrm{ESI}=\frac{\mathrm{HE}-\mathrm{HC}}{\mathrm{HE}} \mathrm{X} 100 \rightarrow \mathrm{Eq} .1
$$

where $\mathrm{HC}$ is the height of cream layer and $\mathrm{HE}$ is the initial emulsion height. 


\section{Rheology study}

The rheological properties of gels were evaluated using a Rheolab MC1 rheometer (Anton Paar, Österreich, Austria) at room temperature. The mode of measurement was viscometry sweep, employing cylinder-cone method with a gap of $0.1 \mathrm{~mm}$. Two rheograms were obtained by plotting apparent viscosity against shear rate and shear stress against shear rate. The rheological data obtained were fitted to the power law model as shown in the following equation.

$$
\tau=\mathrm{K} \dot{\gamma}^{n} \rightarrow \text { Eq. } 2
$$

In which ${ }^{\tau}$ is the shear stress, $\dot{\gamma}$ is the shear rate, $\mathrm{K}$ is the consistency index $\left(\mathrm{Pa}_{\mathrm{s}} \mathrm{s}^{\mathrm{n}}\right)$ and $\mathrm{n}$ is the flow behavior index.

\section{Drug loading and entrapment efficiency}

The drug loading and entrapment efficiency of phenytoin in lipid emulsions were determined using a high-performance liquid chromatography (HPLC) method (Teo et al., 2015). Aliquots of samples were dispersed in the mobile phase of acetonitrile and ultrapure water (50:50). A sample volume of 20 $\mu \mathrm{L}$ was injected at a flow rate of $1.0 \mathrm{~mL} / \mathrm{min}$. The test system used was a reverse phase HPLC system (1200 series, Agilent Technologies) equipped with a pump, injector valve with $20 \mu \mathrm{L}$ sample loop, ZORBAX Eclipse Plus C-18 analytical column (250 $\mathrm{mm} \times 4.6 \mathrm{~mm}, 5 \mu \mathrm{m}$ particles), and UV detector with the data processor (Chem Station Software). A standard curve of peak area against the concentration of phenytoin was plotted and the drug content of each sample was determined by comparison with the standard curve. The following equations were used to calculate the percentage of drug loading and encapsulation efficiency of the emulsions.

$$
\text { Drug loading }(\%)=\frac{\text { Total weight of drug }}{\text { Total weight of sample }} \times 100 \% \rightarrow \text { Eq. } 3
$$

Entrapment efficiency (\%)

$$
=\frac{\text { Actual drug loading }}{\text { Theoretical drug loading }} \times 100 \% \rightarrow \text { Eq. } 4
$$

\section{In vitro drug release study}

The drug release of phenytoin from gel formulations was investigated using Franz diffusion cells (PermeGear, USA). About $1 \mathrm{~g}$ of gel formulation was placed on a $0.45 \mu \mathrm{m}$ pore size, cellulose acetate membrane in the donor compartment. The receptor compartment was filled with $14 \mathrm{~mL}$ of ethanol $96 \%$ which was maintained at $32 \pm 0.5^{\circ} \mathrm{C}$. Aliquots of the receptor medium were withdrawn and replaced with the same amount of fresh receptor medium at time intervals of $1,2,4,6,8$, and 12 hours. The withdrawn aliquots were analyzed by ultraviolet-visible spectrophotometer (Perkin-Elmer, Malaysia) at $240 \mathrm{~nm}$ wavelength. The cumulative percentage of drug release was calculated and plotted against time. Franz diffusion cell study for each formulation was conducted in triplicates and the averages were reported. The release characteristics of gel formulations were further determined by fitting the release data to the following equations of zero-order, first-order and Higuchi models which are given by the following equations.

$$
\begin{gathered}
\mathrm{Q}=\mathrm{k}_{0} t \rightarrow \text { Eq. } 5 \\
\operatorname{In}\left(\mathrm{Q}_{0}-\mathrm{Q}\right)=\operatorname{In} \mathrm{Q}_{0}-\mathrm{k}_{1} t \rightarrow \text { Eq. } 6 \\
\mathrm{Q}=\mathrm{k}_{2} \sqrt{t} \rightarrow \text { Eq. } 7
\end{gathered}
$$

where $\mathrm{Q}_{0}$ is the initial amount of drug, $\mathrm{Q}$ is the amount of drug released at time $t, \mathrm{k}_{0}, \mathrm{k}_{1}$ and $\mathrm{k}_{2}$ are the rate constants for zeroorder, first-order and Higuchi models respectively.

\section{Statistical analysis}

In vitro drug release data of different lipid gel formulations were subjected to multiple t-test using Microsoft Excel 2013. The t-test was performed to find out if there was any significant difference in the release pattern of groups of nanoemulgel and cream-gel; nano-emulgel and macro-emulgel; creamgel and macro-emulgel, over the release intervals of 12 hours. Analysis outcomes showing $\mathrm{P}<0.05$ were considered statistically significant.

\section{RESULTS AND DISCUSSIONS}

\section{Physicochemical properties of lipid emulsions}

Three oil-in-water $(\mathrm{O} / \mathrm{W}) \mathrm{CKO} / \mathrm{SBO}$ emulsions, namely nano-emulsion, macro-emulsion and cream were formulated with the same ingredients as listed in Table 1 but differed in droplet size. From Table 1, the oil-to-surfactant ratio of 1:9 was apparently needed to maintain the stability of the emulsions especially with the presence of the long hydrocarbon chain length of SBO in the lipid blends. This is because oil with longer hydrocarbon chain length would exert higher interfacial tension against the aqueous phase, thus leading to high physicochemical stress. Though oils with long hydrocarbon chains like SBO is difficult to be emulsified compared to medium-chain triglycerides like CKO, the solubilizing capacity of oil for lipophilic moiety increases with the chain length of oil. Hence, the formulation of CKO/SBO blends is a compromise between the solubilizing potential and ability to facilitate the formation of emulsion for entrapping drugs. Previous studies have shown that inclusion of highly saturated mediumchain triglycerides (MCT) into long-chain triglycerides (LCT) yields more stable emulsions than pure LCT by avoiding droplet coalescence (Tamilvanan and Benita, 2004). Table 2 shows the physico-chemical properties of the emulsions. Particle size measurement indicated that PIT method can produce clear and transparent nano-emulsion with a droplet size of $11.4 \mathrm{~nm}$. The formation of finely dispersed oil droplets took place when the system crosses a point of zero spontaneous curvature followed by minimal surface tension during the rapid cooling stage in PIT (Ng et al., 2014). 
Table 1: Formulations of lipid emulsions.

\begin{tabular}{|c|c|c|c|c|c|c|}
\hline \multirow{2}{*}{ Emulsion } & \multicolumn{4}{|c|}{ Percentage weight per weight $(\%$ w/w) } & \multirow{2}{*}{ Oil-to-surfactant ratio } & \multirow{2}{*}{ Oil-to-oil ratio } \\
\hline & CKO & SBO & Tween 80 & Water & & \\
\hline CKO/SBO & 1.4 & 0.6 & 18 & 80 & $1: 9$ & $70: 30$ \\
\hline
\end{tabular}

Table 2: Physico-chemical properties of lipid emulsions.

\begin{tabular}{|c|c|c|c|c|}
\hline Emulsion & Droplet size (nm) & Zeta potential (mV) & pH & Visual observation \\
\hline Nano-emulsion & $11.4 \pm 0.01$ & $-2.90 \pm 0.01$ & $6.6 \pm 0.01$ & Clear and transparent \\
\hline Macro-emulsion & $595.6 \pm 0.02$ & $-0.02 \pm 0.03$ & $6.5 \pm 0.01$ & Translucent white \\
\hline Cream & $167.9 \pm 0.03$ & $-1.45 \pm 0.01$ & $6.6 \pm 0.02$ & Translucent \\
\hline
\end{tabular}

Table 3: Emulsion stability indices of lipid emulsions.

\begin{tabular}{|c|c|c|c|c|c|c|c|c|}
\hline \multirow{2}{*}{ Emulsion } & \multicolumn{8}{|c|}{ ESI $(\%)$ per cycle } \\
\hline & $\mathbf{0}$ & 1 & 2 & 3 & 4 & 5 & 6 & 7 \\
\hline Nano-emulsion & 100 & 100 & 100 & 100 & 100 & 100 & 100 & 100 \\
\hline Macro-emulsion & 100 & 100 & 100 & 94.8 & 84.5 & 72.7 & 61.0 & 50.2 \\
\hline Cream & 100 & 100 & 100 & 100 & 100 & 100 & 98.6 & 98.7 \\
\hline
\end{tabular}

All emulsions exhibited zeta potentials within -2.90 to $0.02 \mathrm{mV}$ which was due to the presence of a non-ionic surfactant, Tween 80 , in which the negatively charged oil droplets were resulted from the absorption of hydroxyl ions on the droplet surface. It was noticed that the $\mathrm{pH}$ values were in the range of 6.5 6.6, which implies that these emulsions would not induce irritations or alterations of the cutaneous tegmentum if applied topically on the skin (Lucero et al., 1994). Table 3 summaries the emulsion stability indices of lipid emulsions. From Table 3, nanoemulsion indicated excellent thermodynamically stable with $100 \%$ ESI and no sign of phase separation throughout the heating/cooling test for seven cycles. Cream presented relatively good stability with no significant phase separation up to $5^{\text {th }}$ heating/cooling cycles but macro-emulsion exhibited the poorest emulsion stability with $100 \%$ ESI up to $2^{\text {nd }}$ heating/cooling cycles. This suggests that stable lipid emulsions could be formulated with oil droplets in nano-size range. The utilization of high amount of non-ionic surfactant Tween $80(18 \% \mathrm{w} / \mathrm{w})$ was necessary for all formulations to reduce the interfacial tension between the two phases and form sufficient surfactant films around the oil phase droplets to prevent coalescence. Furthermore, surfactants of high HLB like Tween 80 was reported to increase the permeability of the drug by loosening the tight junctions around the structural organization of membrane components (Gurram et al., 2015).

\section{Rheological behavior of lipid gels}

Gels are three-dimensional colloidal semisolid formulations having better percutaneous drug absorption and solubilization. Topical gel formulations have a high patient acceptability due to their favorable properties like easily spreadable, long shelf life, greaseless and easily removable. In this study, Aristoflex ${ }^{\circledR}$ AVC was added in all topical gel formulations to increase the viscosity of the medium, which helps create and maintain the suspension of oil droplets within the medium. The addition of Aristoflex ${ }^{\circledR}$ AVC in both nano-emulsion and macroemulsion has successfully modified the fluidity of the emulsions to produce emulgels of higher viscosity. Aristoflex ${ }^{\circledR}$ AVC was also added to the cream to produce cream-gel which has pleasant skin feel like creamy without tackiness. This cream-gel is also expected to have lower irritation potential compared to the conventional cream formulation. From Fig. 1, the gel systems of all topical formulations were found to exhibit non-Newtonian fluid behavior, showing no linear proportionality between shear rate and shear stress. A plot of viscosity as a function of shear rate (Fig. 2) demonstrates that the apparent viscosities of the gel formulations decreased gradually with increasing shear rate. This might due to the deformation of oil droplets flocculations that had previously aggregated by weak forces took place as the shear rate increased (Rao, 2014). From Fig. 2, the flow behavior also indicated that these gels were shear-thinning or pseudoplastic in nature. These findings were further supported by their flow indices with the magnitude of $n<1$, as shown in Table 4, which implied the degree of their pseudoplasticity.

Table 4 shows that all gel formulations exhibited relatively high yield stress, indicating that these gel formulations were relatively stable towards creaming. This was attributed to the highly cross-linked structure of the Aristoflex AVC polymer which has successfully trapped the oil droplets in the water/polymer matrix. This resulted in changes in the structural features and the rheological properties of the continuous phase, thus opposing the motion and mutual contact of droplets, leading to prolong stability of the formulations. The facts that macroemulgel possessed the highest yield stress implied that oil droplets with bigger size are caged more firmly in the network meshes and thus high shearing force was required to trigger its fluid nature. On the other hand, the nano-emulgel showed the highest flow indices (n) pertaining to its lowest pseudoplasticity and superior mobility which could be contributed by its nano-sized oil droplets. Viscosity is dependent on the concentration, size and shape of the suspended particles and how they interact with the continuous phase (Barnes, 2002). However, there was no clear correlation between particle size and viscosity can be concluded when comparing cream-gel and macro-emulgel with nano-emulgel in terms of their droplet size. Agreement with this can be seen for the trend shown in flow indices (Table 4), viscosity (Fig. 2) and droplet size (Table 2). 


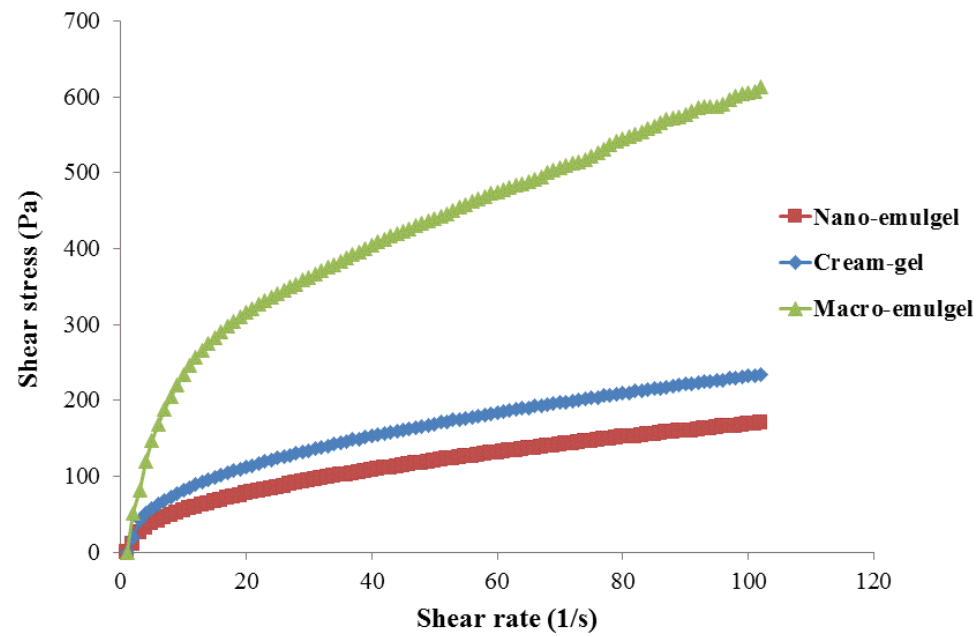

Fig. 1: The flow curves of lipid gels

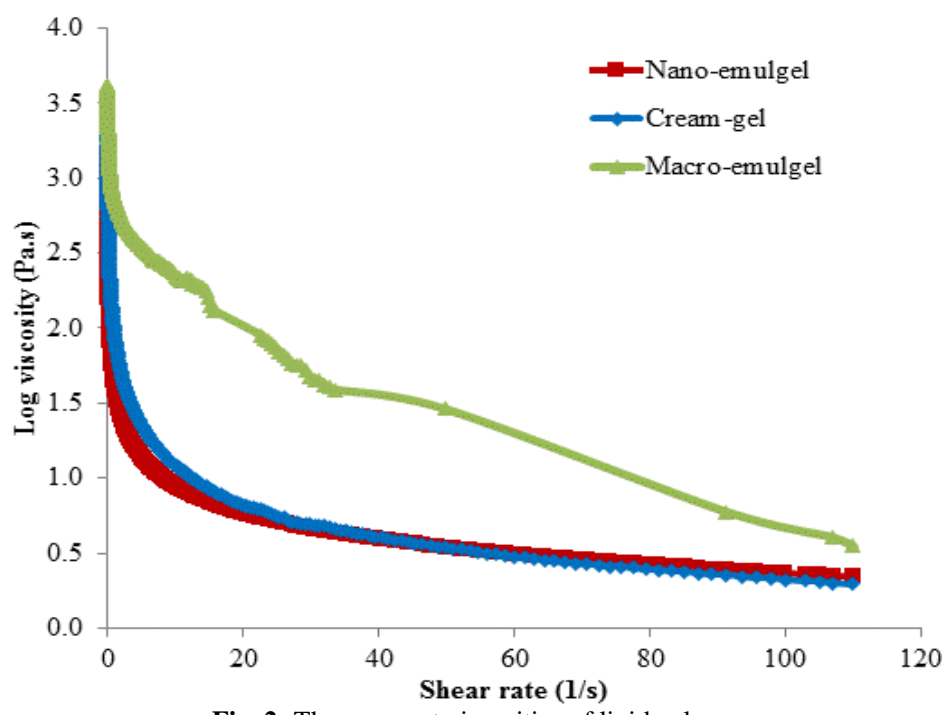

Fig. 2: The apparent viscosities of lipid gels

Table 4: The rheological properties of lipid gel formulations.

Lipid gel Yield stress, $\gamma(\mathbf{P a})$

Nano-emulgel

18.8

Consistency index, K (Pa·s)

35.79

97.39

Cream-gel

34.1

42.39

Flow index, $\mathbf{n}$

0.40

0.39

0.26

\section{Drug loading and entrapment efficiency of lipid emulsions}

In the present study, drug loading and entrapment efficiency of phenytoin were measured in nano-emulsion and macro-emulsion since the preparation of macro-emulsion and cream-gel were rather similar except in the sequence of gelling agent addition. The measured drug loading of phenytoin was recorded as $2 \mathrm{mg} / \mathrm{mL}$ in all formulations, with the encapsulation efficiency was calculated as $98.5 \pm 0.9 \%$ and $95.7 \pm 0.2 \%$ in nanoemulsion and macro-emulsion, respectively. This indicates that phenytoin was successfully encapsulated in the emulsions.

\section{In vitro drug release study of lipid gel formulations}

As shown in Fig. 3, the cumulative percentage of phenytoin released, in a decreasing order was $93.12 \%$ from nano-emulgel, $56.42 \%$ from cream-gel and $51.51 \%$ from macroemulgel. It was apparent that smaller droplet size provides a larger surface area for drug permeation through the membrane at the donor compartment, which resulted in a higher concentration gradient that acts as the driving force for drug permeation. The superior drug release of nano-emulgel compared to macro-emulgel and cream-gel might be also due to the higher lipophilic drug solubilizing capacity of nano-emulgel that leads to an improved topical phenytoin availability. Nano-emulgel with fine droplet size offers advantages over the other two as it improves hydration of stratum corneum, which will increase the drug dermal permeation and skin flux. From Fig. 3, the in vitro release of phenytoin in descending manner (nano-emulgel > cream-gel > macro-emulgel) appeared to be obvious after 2 hours where the cumulative $\%$ of 
drug released of nano-emulgel was significantly dominated over the other two but the drug release pattern of cream-gel and macroemulgel were quite close after 6 hours. Statistical t-test analysis of the data was done to find out whether there was any significant difference in the release profile between the formulations. Nanoemulgel exhibited significant higher cumulative $\%$ of drug release than macro-emulgel except at time intervals of 0 and 1 hour. Drug release profile of nano-emulgel was also found to be significantly greater than those of cream-gel except at the release intervals of 0 , 1 and 2 hour. It was found that the drug release of cream-gel was only significantly higher than macro-emulgel at the first 2 and 4 hours but there was no statistically significance detected between them following the remaining release time intervals as the calculated $\mathrm{P}$ value was greater than 0.05 . This indicated that the release profiles of both cream-gel and macro-emulgel were similar after 6 hours of release intervals. This indicated that there were two phase of phenytoin release profiles for both cream-gel and macro-emulgel. Initially, macro-emulgel with higher viscosity exhibited slower phenytoin released than cream-gel from 0 to 6 hours. When the viscosity is higher, the density of the gels' microstructure increased which limits the area of movement for phenytoin, thus leading to a slower release of phenytoin as observed in macro-emulgel formulation. Nie et al. has demonstrated that faster drug release rate could be achieved from the formulation with low viscosity formulation than that from the high viscous formulation (Nie et al., 2011). However, from Fig. 3, it was also found that the phenytoin release practically stop after 6 hours from both cream-gel and macro-emulgel.
Thus, it is proposed that phenytoin with highly hydrophobicity has a high affinity for the lipid gel formulations, particularly those with larger droplet size or smaller surface area for drug permeation. This is because for mass transfer to take place, the drug must have preferably lesser affinity for the vehicle in order to maximizing the thermodynamic leaving potential. Therefore, this further substantiate the importance of the nano-emulgel formulation in the delivery of topical phenytoin. The release characteristics of lipid gel formulations were further identified by fitting the release data into the equations of zero-order, first-order and Higuchi models. From Table 5, the best-fit release of these three formulations followed the first-order model with $\mathrm{R}^{2}>0.95$. Therefore, the drugs released from the three formulations were concluded as drug concentration dependence.

\section{CONCLUSION}

Three lipid-based gels, namely nano-emulgel, macroemulgel and cream-gel were successfully developed from $\mathrm{CKO} / \mathrm{SBO}$ blended at a ratio of 70:30. These gels demonstrated non-Newtonian, shear-thinning behavior with acceptable viscosity range for the topical application. The in vitro drug release study showed that all lipid-based gels were able to sustain the release of phenytoin within 12 hours which followed the first-order kinetic model. Nano-emulgel showed the highest phenytoin release rate of $93.1 \%$. Hence, nano-emulgel with the nano-emulsion droplet size was identified as the most significant lipid-based delivery system for topical delivery of phenytoin.

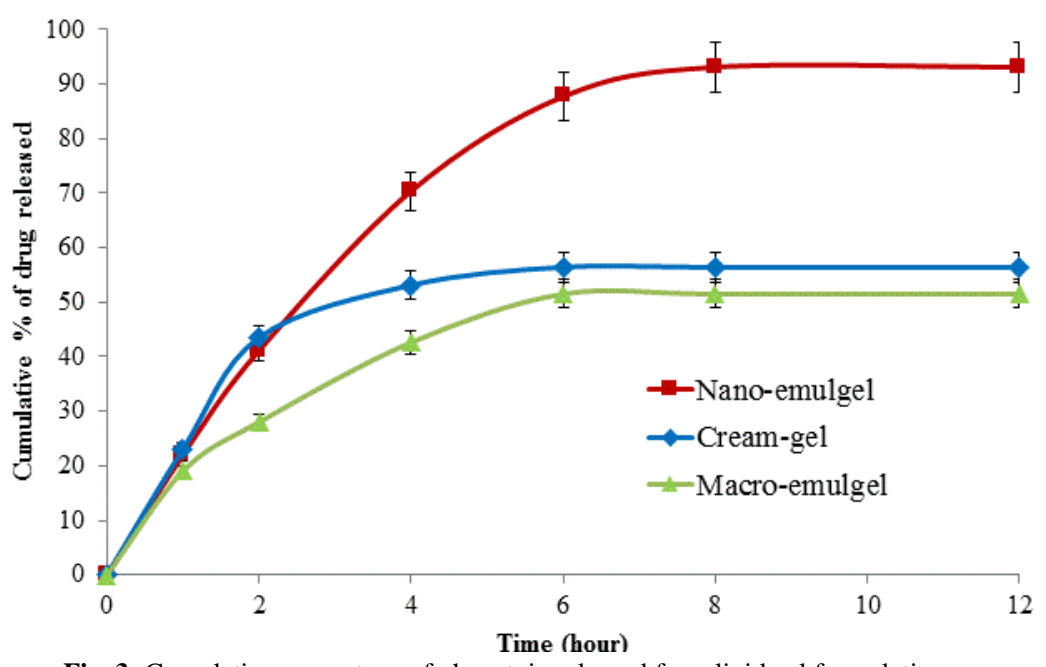

Fig. 3: Cumulative percentage of phenytoin released from lipid gel formulations

Table 5: The release kinetics of different topical phenytoin-lipid gel formulations.

\begin{tabular}{ccccccc}
\hline Formulation & \multicolumn{2}{c}{ Zero-order kinetics } & \multicolumn{2}{c}{ First-order kinetics } & \multicolumn{2}{c}{ Higuchi model } \\
\cline { 2 - 6 } & $\mathbf{R}_{\mathbf{0}}{ }^{2}$ & $\mathbf{K}_{\mathbf{0}}$ & $\mathbf{R}_{\mathbf{1}}{ }^{2}$ & 0.99 & $\mathbf{K}_{\mathbf{1}}$ & $\mathbf{R}_{\mathbf{H}}{ }^{2}$ \\
Nano-emulgel & 0.78 & 13.70 & -0.16 & 0.94 & 40.57 \\
Macro-emulgel & 0.65 & 9.73 & 0.99 & -0.05 & 0.94 & 32.75 \\
Cream-gel & 0.57 & 15.99 & 0.96 & -0.08 & 0.93 & 32.58 \\
\hline
\end{tabular}




\section{CONFLICT OF INTEREST STATEMENT}

Authors declare that they have no conflict of interest in the research.

\section{ACKNOWLEDGEMENT}

The authors thank Mr. Goh Tor Man from MOI Foods Malaysia Sdn. Bhd. for providing lipids used in this study. The authors are indebted to Dr. Koh Rhun Yian from International Medical University for statistical advice.

Financial support and sponsorship: This research is supported in part by International Medical University internal grant (BPI01/11(46)2014) and Malaysia Toray Science Foundation (MTSF) Science and Technology Grant 2014 (IMUR160/2014).

Conflict of Interests: There are no conflicts of interest.

\section{REFERENCES}

Almousilly MM, Abdulrahman LK, Alshmesawy SH, Tawfiq FA. Semisolid dosage form of phenytoin sodium. IRJP, 2012;3(11):71-74.

Bahmani M, Fallahzadeh M, Jowkar F, Khalesi M, Bahri-Najafi R, Namazi M. Can topical phenytoin augment the therapeutic efficacy of PUVA against vitiligo? A double-blind, randomized, bilateral-comparison, placebo-controlled study. J Dermatol Treat, 2011;22(2):106-108.

Barnes HA. 2002. A handbook of elementary rheology. Aberystwyth, Wales: Cambrian Printers.

Bolko K, A. Zvonar A, Gašperlin M. Mixed lipid phase SMEDDS as an innovative approach to enhance resveratrol solubility. Drug Dev Ind Pharm, 2014:40(1):102-109.

Čerpnjak1 K, Zvonar A, Gašperlin M, Vrečer F. Lipid-based systems as a promising approach for enhancing the bioavailability of poorly water-soluble drugs, Acta Pharm, 2013;63:427-445.

Dahan A, Hoffman A. Rationalizing the selection of oral lipid based drug delivery systems by an in vitro dynamic lipolysis model for improved oral bioavailability of poorly water soluble drugs. J Controlled Release, 2008;129:1-10.

Fonseka HFS, Ekanayake SMB, Dissanayake M. Two percent topical phenytoin sodium solution in treating pyodermagangrenosum: a cohort study. Int Wound J, 2010;7:519-523.

Gurram AK, Deshpande PB, Kar SS, Nayak UY, Udupa N, Reddy MS. Role of components in the formation of selfmicroemulsifying drug delivery systems. Indian $\mathrm{J}$ Pharm Sci, 2015;77(3):249-257.

Hokkam E, El-Labban G, Shams M, Rifaat S, El-mezaien M. The use of topical phenytoin for healing of chronic venous ulcerations. Int J Surg, 2011;9(4):335-338.

Jones JJ, Stump MJ, Fleming RC, Lay JO, Wilkins CL. Strategies and data analysis techniques for lipid and phospholipid chemistry elucidation by intact cell MALDI-FTMS. J Am Soc Mass Spectrom, 2004;15:1665-1674.

Lucero MJ, Vigo J, Leon MJ. A study of shear and compression deformations on hydrophilic gels of tretinoin. Int $J$ Pharm, 1994;106(2):125-133.

Mehanna M, Motawaa A, Samaha M. Pharmaceutical particulate carriers: Lipid - based carriers. Natl J Physiol Pharm Pharmacol, 2012;2(1):10-22.

Nie S, Wu J, Liu H, Pan W, Liu Y. Influence of admixed citric acid and physiological variables on the vinpocetine release from sodium alginate compressed matrix tablets. Drug Dev Ind Pharm, 2011;37(8):954962.
Ng S, Lai O, Abas F, Lim H, Tan C. Stability of a concentrated oil-in-water emulsion model prepared using palm olein-based diacylglycerol/virgin coconut oil blends: Effects of the rheological properties, droplet size distribution and microstructure. Food Res Int, 2014;64:919-930.

Prajapati HN, Dalrymple MD, Serajuddin TMA. A comparative evaluation of mono-, di- and triglyceride of medium chain fatty acids by lipid/surfactant/water phase diagram, solubility determination and dispersion testing for application in pharmaceutical dosage form development. Pharm Res, 2012;29:285-305.

Pouton, CW. Formulation of poorly water-soluble drugs for oral administration: Physicochemical and physiological issues and the lipid formulation classification system. Eur J Pharm Sci, 2006;29:278-287.

Rao MA. 2014. Flow and functional models for rheological properties of fluid food. In: Rheology of fluid, semisolid, and solid foods: principle and applications. United States: Springer 27-36.

Rashidi F, Sehhati F, Ghojazadeh M, Javadzadeh Y, Haghsaie $M$. The effect of phenytoin cream in comparison with Betadine solution on episiotomy pain of primiparous women. JCS, 2012;1(2):61-65.

Rhodes RS, Heyneman CA, Culbertson VL, Wilson SE, Phattak HM. Topical phenytoin treatment of stage II decubitus ulcers in the elderly. DICP Ann Pharmacother, 2001;35:675-681.

Rhodes RS, Kuykendall JR, Heyneman CA, May MP, Bhushan A. Stability of phenytoin sodium suspensions for treatment of open wounds. Int J Pharm Compd, 2006;10(1):74-78.

Shaw J, Hughes CM, Lagan KM, Bell PM. The clinical effect of topical phenytoin on wound healing: A systematic review. $\mathrm{Br} J$ Dermatol, 2007;157(5):997-1004.

Shinoda K, Saito H. The stability of O/W type emulsions as functions of temperature and the HLB of emulsifiers: the emulsification by PIT method. J Colloid Interface Sci, 1969;30:258-263.

Shrestha H, Bala R, Arora S. Lipid-based drug delivery systems. J Pharm, 2014;2014:1-10.

Simsek G, Ciftci O, Karadag N, Karatas E, Kizilay A. Effects of topical phenytoin on nasal wound healing after mechanical trauma: An experimental study. Laryngoscope 2014;124:1-6.

Smith RG. Off-label use of prescription medication: A literature review. Wounds, 2010;22(4):78-86.

Shaw J, Hughes CM, Lagan KM, Bell PM. The clinical effect of topical phenytoin on wound healing: A systematic review. $\mathrm{Br} J$ Dermatol, 2007;157(5):997-1004.

Spaia S, Eleftheriadis T, Pazarloglou M, Askepidis N, Ioannidis I, Touboura A, Vayonas G. Phenytoin efficacy in treating the diabetic foot ulcer of a haemodialysis patient. Nephrol Dial Transplant, 2004;19(3):753.

Yadav N, Khatak S, Sara UVS. Solid lipid nanoparticles - A review.

Int J Appl Pharm, 2013;5(2):8-18.

Tamilvanan, S, Benita S. The potential of lipid emulsion for ocular delivery of lipophilic drugs. Eur J Pharm Biopharm, 2004;58:357368.

Teo SY, Rathbone MJ, Coombes AGA, Lee SY, Gan SN. Development and validation of a stability-indicating isocratic reverse phase-liquid chromatography assay for determination of phenytoin in bulk and pharmaceutical formulations. IJPPS, 2015;7(8):258-263.

\section{How to cite this article:}

Lee SY, Pung YY, Khor BK, Kong WE, Tan CT, Teo SY. LipidBased Delivery System for Topical Phenytoin. J App Pharm Sci, 2016; 6 (11): 014-020. 